\title{
Field-response of magnetic vortices in dusty olivine from the Semarkona chondrite
}

Nichols, Claire I. O.; Einsle, Joshua F.; Im, Mi-Young; Kasama, Takeshi; Saghi, Zineb; Midgley, Paul A.; Harrison, Richard J.

Published in:

Geochemistry, Geophysics, Geosystems

Link to article, DOI:

10.1029/2018GC008159

Publication date:

2019

Document Version

Peer reviewed version

Link back to DTU Orbit

Citation (APA):

Nichols, C. I. O., Einsle, J. F., Im, M-Y., Kasama, T., Saghi, Z., Midgley, P. A., \& Harrison, R. J. (2019). Fieldresponse of magnetic vortices in dusty olivine from the Semarkona chondrite. Geochemistry, Geophysics, Geosystems, 20(3), 1441-1453. https://doi.org/10.1029/2018GC008159

\section{General rights}

Copyright and moral rights for the publications made accessible in the public portal are retained by the authors and/or other copyright owners and it is a condition of accessing publications that users recognise and abide by the legal requirements associated with these rights.

- Users may download and print one copy of any publication from the public portal for the purpose of private study or research.

- You may not further distribute the material or use it for any profit-making activity or commercial gain

- You may freely distribute the URL identifying the publication in the public portal 


\section{Field-response of magnetic vortices in dusty olivine from the Semarkona chondrite}

Claire I. O. Nichols ${ }^{1,2}$, Joshua F. Einsle ${ }^{1,3}$, Mi-Young $\operatorname{Im}^{4,5,6}$,

Takeshi Kasama $^{7}$, Zineb Saghi ${ }^{3,8}$, Paul A. Midgley ${ }^{3}$, and

Richard J. Harrison ${ }^{1}$

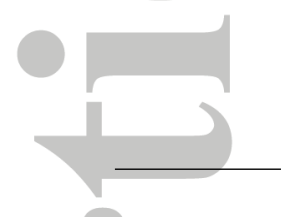

Claire I. O. Nichols, cion2@mit.edu

${ }^{1}$ Department of Earth Sciences,

University of Cambridge, Downing Street,

Cambridge, CB2 3EQ, UK

${ }^{2}$ Department of Earth, Atmospheric and

Planetary Sciences, MIT, Cambridge, MA

02139, USA

${ }^{3}$ Department of Materials Science and

Metallurgy, University of Cambridge, CB3

OFS, UK

${ }^{4}$ Center for X-ray Optics, Lawrence

Berkeley National Laboratory, Berkeley, CA

94720, USA

This article has been accepted for publication and undergone full peer review but has not been through the copyediting, typesetting, pagination and proofreading process, which may lead to differences between this version and the Version of Record. Please cite this article as doi: 10.1029/2018GC008159

(C)2019 American Geophysical Union. All Rights Reserved. 
Abstract. Recent paleomagnetic studies have constrained the strength and longevity of the magnetic field generated by the solar nebula, which has broad implications for the early evolution of the solar system. Paleomagnetic evidence was recorded by nanoscale iron inclusions in olivine crystals in the Semarkona LL 3.0 chondrite. These dusty olivines, have been shown to be credible carriers of ancient magnetic remanence. The small scale of the iron inclusions presents several challenges for defining their fundamental magnetic properties. Here we present the first correlative study of the response of these magnetic structures under applied laboratory fields. Results show that the majority of particles are in a single-vortex state and exhibit stable magnetic

${ }^{5}$ Department of Emerging Materials

Science, DGIST, Daegu, Korea

${ }^{6}$ School of Materials Science and

Engineering, Ulsan National Institute of

Science and Technology (UNIST), Ulsan

44919, Korea

${ }^{7}$ Center for Electron Nanoscopy, Technical

University of Denmark, DK-2800 Kongens

Lyngby, Denmark

${ }^{8} \mathrm{CEA}, \mathrm{LETI}, \mathrm{MINATEC}$ Campus,

F-38054 Grenoble, France

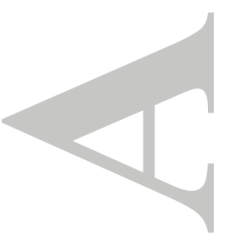

(C)2019 American Geophysical Union. All Rights Reserved. 
behavior in applied fields up to $200 \mathrm{mT}$. Experimental observations using Lorentz microscopy and magnetic transmission X-ray microscopy are shown to compare well with the results of finite-element micromagnetic simulations derived from 3D models of the particles obtained using electron tomography. This correlative approach may be used to characterize the fundamental magnetic behavior of many terrestrial and extraterrestrial paleomagnetic carriers in the single- to multi-vortex size range, which represent the vast majority of stable magnetic carriers in rocks and meteorites.

\section{Plain Language Summary:}

Some of the first solid materials to form in the solar system have been brought to Earth by meteorites. They contain tiny metallic inclusions which record information about the magnetic fields at the earliest stages of our solar system's history. Understanding these magnetic fields, and how they are recorded by metallic particles, provides very important information for understanding how our solar system formed and evolved. We have studied some of these particles to image their magnetic structure using microscopes which allow us to see structures a billionth of a metre in size. We have developed a new technique using X-rays to image how the magnetic structure in these particles changes when we apply different magnetic fields in the laboratory. We have also been able to reproduce our results using computer simulations of the magnetic behavior of the particles. This is the first study that has imaged the magnetic structure of these particles under applied fields. We find that the particles are very stable; even under magnetic fields thousands of

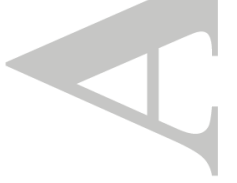

(C)2019 American Geophysical Union. All Rights Reserved. 
times stronger than Earth's, the particles still don't change the magnetic structure they had in the early solar system.

\section{Keypoints:}

- We show the first experimental results demonstrating the stability of dusty olivines under applied magnetic fields.

- We use a novel combination of electron and X-ray microscopy techniques combined with micromagnetic simulations.

- We confirm previous results that dusty olivines are capable of recording magnetic fields from the solar nebula.
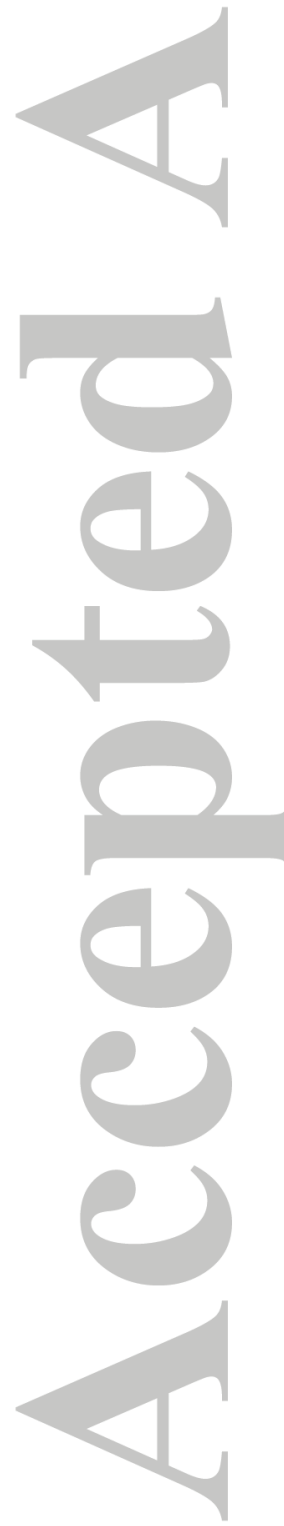

(C)2019 American Geophysical Union. All Rights Reserved. 


\section{Introduction}

The Semarkona meteorite is an LL 3.0 ordinary chondrite, containing some of the most pristine and unequilibrated chondrules. These chondrules contain dusty olivine grains, formed by reduction of olivine in the earliest stages of the solar nebula. These dusty olivines are not thought to have formed by crystallization within the host chondrule, but represent relict grains of an even earlier time in solar system history, prior to chondrule formation [Leroux et al., 2003]. The dusty olivines contain sub-micron sized metallic grains of almost pure Fe kamacite [Uehara and Nakamura, 2006]. The composition of metallic grains considered in this study was measured using scanning transmission electron microscopy elemental maps in previous work [Fu et al., 2014a]. A recent 3D micromagnetic and tomographic study of the Semarkona meteorite concluded that the majority of stable remanence carriers in dusty olivines were vortex and small multidomain states [Einsle et al., 2016]. Significant research has been undertaken to investigate the credibility of these Fe particles as paleomagnetic carriers of solar nebula magnetic fields [Einsle et al., 2016; Lappe et al., 2011; Shah et al., 2018]. The first paleomagnetic measurement of a solar nebula field was captured by dusty olivines from the Semarkona chondrite, with important implications for the formation of the pre-accretionary disk and the first solid material in the solar system [Fu et al., 2014b].

Recent research has also shown that single-vortex (SV) grains can behave like uniaxial single domain (SD) grains, and may in fact be more stable as paleomagnetic recorders [Almeida et al., 2014; Nagy et al., 2017]. This is a significant discovery for all fields of paleomagnetic research, since the vast majority of magnetic grains in rocks fall in the

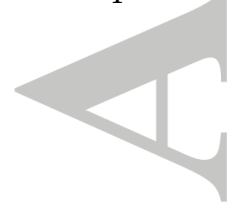

(C)2019 American Geophysical Union. All Rights Reserved. 
vortex to multi-vortex size range from $\sim 0.1-10 \mu \mathrm{m}$ [Roberts et al., 2018]. Off-axis electron holography in the transmission electron microscope (TEM) has directly imaged vortex behavior at a range of temperatures and shows good agreement with micromagnetic simulations. These results suggest that vortex structures are stable recorders of both thermoremanent magnetization, paleodirections and paleointensities [Almeida et al., 2016; Shah et al., 2018].

The aim of this study was to image directly the magnetic response of dusty olivine inclusions under high applied magnetic fields (where high refers to fields on the order of millitesla), and thereby assess the validity of micromagnetic simulations that predict high field stability for these particles. Magnetic imaging in high applied fields is extremely challenging using conventional electron-beam techniques (e.g. electron holography) since strong magnetic fields adversely affect the trajectory of electrons through the microscope. For this reason, previous experiments which image vortex states in natural materials have been performed under zero-field conditions [Shah et al., 2018]. The magnetic soft X-ray transmission microscope at the XM-1 beamline at the Advanced Light Source (Berkeley, California) offers a particularly attractive set up for imaging in applied magnetic fields, since it operates in a photon-in/photon-out regime, therefore magnetic fields may be applied in any orientation without affecting the image. The XM-1 beamline is capable of $15 \mathrm{~nm}$ spatial resolution [Fischer et al., 2006], allowing the behaviour of the smallest magnetic structures to be imaged.

There have been several successful experiments that utilize X-ray magnetic circular dichroism (XMCD) to image vortex core behavior with picosecond time resolution [Choe et al., 2004; Guslienko et al., 2006; Im et al., 2014; Raabe et al., 2005]. This research (C)2019 American Geophysical Union. All Rights Reserved. 
focusses on the application of vortex cores as memory devices and uses permalloy thin films with a thickness of $20 \mathrm{~nm}$. These ultra-thin materials provide excellent imaging contrast. Natural samples, on the other hand, are much thicker and more heterogeneous, posing several technical challenges for successful imaging. Here, we show the first results for the response of natural dusty olivines to applied fields of up to $\sim 200 \mathrm{mT}$ at room temperature, using magnetic soft X-ray transmission microscopy (MTXM). Results are presented for five particles from a dusty olivine grain from the Semarkona chondrite (particle dimensions are given in Table 1). The motion of vortex structures in applied fields is found to be in good agreement with micromagnetic simulations, based on the exact morphology of the particles obtained from electron nanotomography.

A correlative approach is used to interrogate the fundamental properties of nanoscale magnetic particles under strong applied magnetic fields (Figure 1). We demonstrate the advantage of combining a variety of experimental techniques to build a comprehensive data set for specific particles within a sample. The potential for realistic 3D reconstructions and simulations is also shown, allowing time-consuming experimental procedures to be supplemented by computational results.

\section{Materials and Methods}

\subsection{Samples}

Iron particles from a dusty olivine grain, sample DOC5 described by Fu et al. [2014b], were extracted from a chondrule from the Semarkona LL3.0 ordinary chondrite [Einsle et al., 2016; Fu et al., 2014b]. Five iron particles formed the focus of the study, the dimensions of which are summarised in Table 1. Three particles were significantly more elongate that the other two, offering the opportunity to investigate the effects of shape (C)2019 American Geophysical Union. All Rights Reserved. 
anisotropy. The dusty olivine grain was mounted in epoxy on a quartz disc. The disc was subsequently mounted on an scanning electron microscope (SEM) stub and carbon coated. The olivine grain needed to be as thin as possible in order to carry out MTXM. A lamella was prepared using the focussed ion beam (FIB) in a lift-out technique on the FEI Helios Nanolab DualBeam in the Department of Materials Science and Metallurgy, University of Cambridge. The center and one edge of the sample were gradually thinned using a stable window configuration (Figure 2). Within the stable window, the sample was gradually thinned into a wedge shape, following the methodology described by Schaffer et al. [2012]. The sample was then mounted on an omniprobe grid with platinum deposition.

\subsection{Lorentz Microscopy}

Lorentz microscopy in the TEM was conducted at the Center for Electron Nanoscopy, Technical University of Denmark. Images were acquired using an FEI Titan 80-300 TEM with a field-emission-gun (FEG) operated at $300 \mathrm{keV}$. Electrons are transmitted through the sample and deflected by its magnetization. The direction of magnetization can be determined by using Fresnel imaging, whereby the electron beam is over-focused onto the specimen, leading to bright or dark contrast. The contrast is generated by the in-plane magnetization of the sample causing deflection of the electron beam due to the Lorentz force [Kasama et al., 2009]. Particles with complex internal structure were then selected for further study.

\subsection{Full-field soft X-ray transmission microscopy}

Selected samples were measured using full-field soft X-ray transmission microscopy at beamline 6.1.2 (XM-1) at the Advanced Light Source (Lawrence Berkeley National Lab-

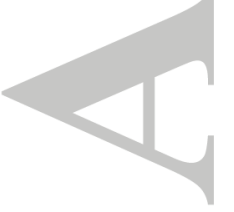

(C)2019 American Geophysical Union. All Rights Reserved. 
oratory, California). This beamline offers a simple set up: X-rays are emitted from a bending magnet source and focused using two Fresnel zone plates placed at the focal points in front and behind the sample. The sample is mounted on an omniprobe grid, which is taped to the corner of a flat, metal window and then screwed to the sample stage. Samples were imaged under magnetic fields in both in-plane (IP) and out-of-plane (OOP) configurations (Figure 3) . The sample was mounted on a holder which sits at an angle of $60^{\circ}$ to the direction of transmission [Fischer et al., 2001]. In the IP configuration, a magnetic field is applied parallel to the sample surface (Figure 3a,b) and in the OOP configuration the applied field is perpendicular to the sample surface (Figure 3c). Magnetic fields are applied using two magnetic pole pieces. The applied field is generated by applying a current of up to $1 \mathrm{~A}$ in either direction across the pole pieces. A $1 \mathrm{~A}$ current corresponds to a magnetic field of $\sim 100 \mathrm{mT}$ and $\sim 300 \mathrm{mT}$ for the IP and OOP configuration, respectively. Magnetic contrast is achieved using X-ray magnetic circular dichroism (XMCD). The bending magnet alters the polarity of the X-rays; by changing the angle of the X-rays to the orbital plane by a few milliradians, $70 \%$ polarisation is achieved. The polarity above the beam center is the opposite of that below the beam center, so by selecting different regions of the X-ray beam, opposite polarisations were acquired. Due to inhomogeneity in the beamline, opposite polarizations are not the exact reverse of each other, therefore variation in contrast between opposite polarizations cannot be quantified. Changes in the magnetic signal are also very weak relative to the X-ray absorption of the Fe particles as a whole. In order to generate a visible, quantifiable magnetic contrast, two images acquired in a magnetic field applied in opposite directions (e.g., $10 \mathrm{mT}$ and $-10 \mathrm{mT}$ ), but with the same polarization are divided (Figure 4). This gives an intensity

(C)2019 American Geophysical Union. All Rights Reserved. 
of unity anywhere where magnetic contrast has not changed and small variations (mostly $5 \%$ ) where magnetic structures have shifted (Figure 1). This method therefore produces magnetic contrast generated by the difference in magnetization between the two applied fields. Forty images were acquired for each applied field direction. Each pair of images is divided and then the average of forty images is taken in order to minimize noise (Figure 5). Only magnetic contrast generated in the center of the particles is considered for further analysis. High contrast regions around the edges of the particles are artifacts which are present prior to any image processing.

\subsection{HAADF-STEM Tomography}

High angle annular dark field scanning transmission electron microscope (HAADFSTEM) tomography was carried out using an FEI Tecnai F20 FEG TEM in the Department of Materials Sciences and Metallurgy, University of Cambridge. HAADF imaging has the advantage of being less affected by diffraction contrast and producing an image that is essentially monotonic with thickness and is only sensitive to changes in composition, therefore projection images accurately reflect the genuine particle structure [Midgley and Dunin-Borkowski, 2009; Midgley and Weyland, 2003]. Images were acquired every degree between $75^{\circ}$ along a single tilt axis, defined by a protective Pt strip. This experimental set up resulted in a missing wedge of $30^{\circ}$. The Pt strip also partially obscures the two Fe nanoparticles of interest. Images were aligned using FEI's Inspect3D. We reduce reconstruction artefacts due to the missing wedge and the overlapping of the Pt strip at high angles by using a compressive sensing (CS) algorithm [Leary et al., 2013; Saghi et al., 2011].

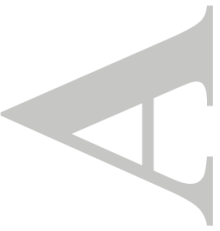

(C)2019 American Geophysical Union. All Rights Reserved. 


\subsection{Micromagnetic Simulations}

Micromagnetic simulations were used to investigate the observed MTXM behaviour of the particles. The shape and size of the particles were accurately reconstructed from the tomography data. The tomographic reconstructions were smoothed using the image processing package Image J and converted to a triangular surface mesh. The software package $\mathrm{CuBit}$ was then used to convert to a tetrahedral volume mesh. Tetrahedral mesh nodes are distributed at $5 \mathrm{~nm}$ intervals throughout the particle. Although this is slightly larger than the exchange length for Fe $(3.4 \mathrm{~nm})$, a denser node distribution is too computationally expensive. Nodes at $5 \mathrm{~nm}$ intervals provide acceptable resolution for the simple simulations presented here.

Micromagnetic simulations were performed using Micromagnetic Earth Related Rapid Interpreted Language Laboratory (MERRILL) [Conbhui et al., 2018]. A finite-element method and boundary-element method were used to solve for the magnetic scalar potential inside the particle, in order to calculate the demagnetizing energy of the system. The Fe particles were simulated under applied fields in the same orientation as those applied during the MTXM experiment. Simulations were carried out between -1000 mT and 1000 mT in steps of $10 \mathrm{mT}$. At each step, the simulation finds the lowest energy state, taking into account exchange, cubic anisotropy, magnetostatic and demagnetizing energies. Material properties for $\mathrm{Fe}$ at $20{ }^{\circ} \mathrm{C}$ were assumed: saturation magnetization, $\mathrm{M}=1715 \mathrm{kA} \mathrm{m}^{-1}$; exchange energy, $\mathrm{A}=2 \times 10^{-11} \mathrm{~J} \mathrm{~m}^{-1}$; and cubic anisotropy, $\mathrm{K}_{1}=48 \mathrm{~kJ} \mathrm{~m}^{-3}[$ Muxworthy and Williams, 2015]. At the end of each applied field step, magnetic moments within the sample were randomly rotated by up to 30 ; this was used as the starting point for the

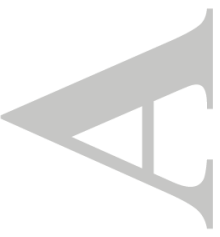

(C)2019 American Geophysical Union. All Rights Reserved. 
next applied field step to avoid the simulation terminating in a local energy minimum. These simulations give a 3D representation of the magnetic behavior of the particle.

In order to directly compare the simulations with the MTXM data, representative 2D projections were calculated. Tetrahedral volume meshes were interpolated onto a regularly structured grid of $100 \times 100 \times 100$ nodes. The magnetic moment at each node in the structured grid is the contribution from all of the surrounding nodes in the tetrahedral volume mesh. The entire stack is then averaged in the z-direction to give a $100 \times 100$ 2D projection of the Fe particle. In the MTXM experiments the Fe particles are highly absorbing, with only $20 \%$ of the photons being transmitted. Therefore, a background value is added to the simulated projection to represent this absorption. An image is then generated for qualitative comparison with the MTXM data.

\section{Results}

\subsection{Particle Characterisation}

In order to compare experimental results with micromagnetic simulations, accurate 3D morphologies of the particles measured using Lorentz microscopy and MTXM were reconstructed using STEM nanotomography (Figure 6). The dimensions of each measured particle are shown in Table 1. Their shape in cross section varies from approximately circular to elliptical with a maximum aspect ratio of 1.3 (Particle 4 ). The top and bottom of each particle is flattened in order to make them thin enough for MTXM.

The morphology of each particle controls the magnetic behaviour it exhibits. Particles 2 and 5 have the most equant morphologies. Their magnetization is in a single vortex state, with the vortex core positioned perpendicular to the flattened top and bottom of each

particle. Particle 1 and Particle 3 are slightly elongate, and have a vortex core aligned (C)2019 American Geophysical Union. All Rights Reserved. 
the major axis of each particle. Particle 4, which has the highest aspect ratio, shows an internal Kittel structure. The domain walls divide the particle into four quadrants forming a Kittel closure structure [Kittel, 1946]. Since all of the particle are similar in size, these changes in internal magnetic structure can be attributed to their shape.

\subsection{Imaging the magnetic structure of nanoscale Fe particles}

Over-focussed (Figure 7a) and under-focussed (Figure 7b) Lorentz images were acquired for Particles 4 and 5. In the over-focused image, a bright region of contrast runs along the center of the long-axis of Particle 4. Particle 5 has a dark center and will be referred to as the Vortex particle due to its single vortex magnetic structure. The alternate contrast observed in the two particles demonstrates that the magnetisation of each of the particles rotates in opposite senses. The under-focused image confirms these observations; Particle 4 has a dark center, while Particle 5 has a bright center. The high-resolution contrast sharply defines the interior magnetic structure of these particles.

The same particles that were imaged using Lorentz microscopy were then imaged using MTXM (Figures 7c and 8). Using this method, the lateral shift of the domain wall and/or vortex core in response to the oppositely applied magnetic field is seen as a paired bright/dark feature, as shown schematically in Figure 4. The particles were imaged under an increasing out-of-plane (OOP) applied magnetic field from 20 to $200 \mathrm{mT}$ (Figure 3d). As the applied field was increased, the observed contrast within the particles increased, corresponding to larger shifts of the interior magnetic structure. The internal structure in Particle 5 shifts in the opposite direction to Particle 4, causing the light and dark contrast

to be reversed. This is consistent with results from Lorentz microscopy.

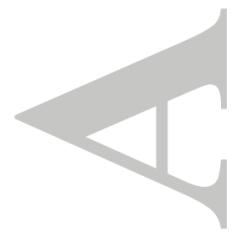

(C)2019 American Geophysical Union. All Rights Reserved. 
To investigate the response of the internal magnetic structure to the direction of the applied magnetic field, three more particles (Particle 1, Particle 2 and Particle 3) were imaged in one IP (Figure 3b) and two OOP orientations (Figure 3d). The different magnetic response to each applied field direction was observed in the MTXM contrast (Figure 9). The IP applied field causes the largest shift of the vortices, since it is applied almost perpendicular to each vortex core. For the two OOP applied field directions, there is also an IP component since the sample is mounted at a $30^{\circ}$ angle to the direction of the applied field. The response of the particles depends on their morphology relative to the direction of the IP component of the applied field (direction of the IP applied field is shown in Figure 9). A larger shift was observed when the IP component was perpendicular to the elongate axis of the particles. Particle 2, which has the most equant morphology was the most stable; the single vortex structure only experienced small shifts, even under applied fields of $200 \mathrm{mT}$.

All of the changes in magnetic structure are confined toward the center of the particles, most of which exhibit single vortex cores. For all applied field directions and particles, no domain wall annihilation has occurred at $200 \mathrm{mT}$, suggesting they are still extremely stable under applied fields of this strength.

\subsection{Comparing experimental results with micromagnetic simulations}

The nanotomography of each particle was converted to a mesh and used with MERRILL to generate 3D micromagnetic simulations of particle behavior under varying applied magnetic fields. The $3 \mathrm{D}$ simulations were then converted to a $2 \mathrm{D}$ projection for direct comparison with the MTXM experimental data. Figures 8 and 9 show experimental and simulated MTXM images. In all cases the simulations are in good qualitative agreement, (C)2019 American Geophysical Union. All Rights Reserved. 
although slightly larger shifts are calculated for a given applied field strength. Results from micromagnetic simulations can be used to begin to interpret the response of the particles to applied magnetic fields. The hysteresis loops shown in Figure 9 demonstrate how the change in applied field direction and particle morphology influence magnetic behaviour. Vortices are annihilated (i.e., the vortex is driven out of the particle as it approaches saturation) at lower applied fields in the IP configuration, or when the inplane component of the OOP field is aligned along the short axis of elongate particles.

Paraview [Ahrens et al., 2005; Ayachit, 2016] was used to visualise how the change in contrast in the processed MTXM images corresponds to the actual shift in magnetic structure. Profiles of simulated MTXM intensity were taken across the particles (Figure 10). The profiles are asymmetric between the light and dark regions. This implies that the shift in magnetic structure is small, i.e. the change in magnetic structure for positive and negative applied fields overlaps. The magnitude of the MTXM signal increases as the particles become more strongly magnetized.

Simulations were run for a larger range of applied field strengths than were investigated experimentally, ranging between $\pm 1 \mathrm{~T}$ (Figure 9). The applied field strengths at which vortices are annihilated are shown in Table 2 and are found to exceed $200 \mathrm{mT}$ in all cases.

\section{Discussion}

Results show the first direct experimental observations of the magnetic response of vortex states in natural samples to an applied external magnetic field. Fe particles in dusty olivine grains are found to be stable under strong applied fields up to $200 \mathrm{mT}$.

This is in good agreement with recent studies suggesting single-vortex grains may in fact be more stable than SD particles [Nagy et al., 2017]. In the following, we discuss the (C)2019 American Geophysical Union. All Rights Reserved. 
various experimental techniques used to image the magnetic structure of the particles and the merits of combining multiple techniques with micromagnetic simulations to fully characterize the observed behavior.

Lorentz microscopy images magnetic contrast within particles using the deflection experienced by electrons as they pass through a region of magnetic induction [Chapman and Scheinfein, 1999]. Figure 7 shows the high resolution magnetic structure within the particles. The Lorentz images are much higher resolution than the equivalent MTXM images, demonstrating the superiority of the Lorentz method for the direct imaging of the magnetic structure. The magnetic structure of the Particle 4 is revealed in the overfocused image (Figure 7a), while the magnetic structure of Particle 5 is best seen in the under-focused image (Figure 7b). This is consistent with the opposite response of each of the particles to an applied magnetic field, shown by the opposite change in contrast in the MTXM image (Figure 7c). Although Lorentz microscopy provides better contrast, imaging in the presence of a large magnetic field is experimentally highly challenging.

MTXM has an advantage over Lorentz microscopy in allowing direct imaging of the response of magnetic structure to a large applied field. The results presented here demonstrate a proof-of-concept that natural, nanoscale particles can be imaged with a resolution high enough to monitor their response under varying magnetic fields. This technique is already well established for vortex dynamics in permalloys [Im et al., 2014; Kasai et al., 2008]. Several experimental challenges were encountered specific to the imaging of natural samples. Because natural samples are thicker and more heterogeneous than idealized permalloys, the magnetic signals from the particles are very small, particularly compared to the high X-ray absorption of the Fe particle as a whole (Figure 4). In order to visualize (C)2019 American Geophysical Union. All Rights Reserved. 
magnetic structure within the particles, one image must be divided by another in different applied fields. This removes the large absorption step from the Fe particle, which does not change with varying applied field (Figure 5). Magnetic contrast generates a $<5 \%$ change in intensity, for equal and opposite applied fields up to $\sim 200 \mathrm{mT}$.

In order to compare experimental results to micromagnetic simulations, the exact morphology of the particles must be known. We were able to accurately constrain the morphology of the studied iron particles in the size range $\sim 200-600 \mathrm{~nm}$ using STEM tomography. This allows the influence of a precise morphology on the magnetic response of particles to be simulated under various applied field conditions. This also highlights the merits of using this method to investigate the response of more complex morphologies, which are often encountered in natural samples, e.g. dendrites, are clear [Shaar and Tauxe, 2013; Lascu et al., 2018].

$3 \mathrm{D}$ reconstructions highlight the flattened top and bottom of the particles. Particles have to be $<200 \mathrm{~nm}$ thick in order to transmit X-rays. This means that the particles studied here, which have a diameter exceeding $200 \mathrm{~nm}$ (Table 1), cannot be imaged with their natural morphology and must be made planar, hence altering their magnetic behavior. This is a problem for any experimental technique requiring transmission through extremely thin samples e.g., electron holography [Shah et al., 2018]. New imaging methods are currently being developed which use hard X-rays. These higher energy X-rays allow high resolution, temporally-resolved imaging in applied fields for thicker samples with their natural morphology [Falch et al., 2017]. There has been one successful study using hard X-rays to image the 3D magnetization of a synthetic sample [Donnelly et al., 2017].

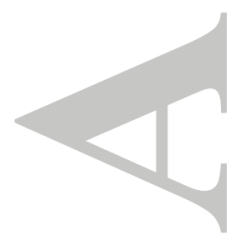

(C)2019 American Geophysical Union. All Rights Reserved. 
This is extremely promising for future successful imaging of the natural morphology of magnetic carriers.

The micromagnetic simulations are found to be in good agreement with the experimental data. Some small discrepancies are observed between the MTXM and micromagnetic images, however these are attributed to the experimental challenges of acquiring and processing MTXM data. This suggests that the simulations are reliable enough to extend the results to higher applied fields and varying field directions, and could also be used to simulate the natural morphology of particles without the need to plane-off their tops and bottoms. Simulations can also be used to define rock magnetic properties for specific samples. When considering nanoscale inclusions, such as those presented here, experimental methods are extremely challenging, expensive and time consuming. Therefore the ability to enhance experimental data with realistic simulations is important for the success and scope of future nanomagnetic studies.

Another advantage of micromagnetic simulations is the diversity with which the results can be presented. 3D reconstructions of the magnetic behavior within an individual particle are invaluable for fully interpreting their response (Figure 10c). Experimental images may also be reproduced, such as those acquired during MTXM experiments (Figures 8 and 9). Results from electron holography have also been simulated from micromagnetic simulations [Almeida et al., 2014; Conbhuí et al., 2016].

Experimental and simulated MTXM results suggest that dusty olivine particles are stable in strong applied fields of at least $200 \mathrm{mT}$. This applied field is much stronger than any magnetic field the samples were likely to encounter in the solar system (most planetarystrength magnetic fields are a few tens to hundreds of microtesla). Our results support (C)2019 American Geophysical Union. All Rights Reserved. 
previous experimental studies and simulations that have demonstrated the thermal stability of dusty olivines over timescales equivalent to the age of the solar system [Almeida et al., 2016; Nagy et al., 2017; Shah et al., 2018; Nagy et al., 2019], together, these results suggest that dusty olivines are excellent paleomagnetic recorders.

\section{Conclusions}

A correlative approach is presented for the direct investigation of nanoscale magnetic properties in dusty olivine particles from the Semarkona meteorite. Lorentz microscopy was used to provide high resolution images of the internal magnetic structure of nanoscale particles. Selected particles were then imaged using MTXM to investigate their response under strong applied magnetic fields. STEM tomography was subsequently used to create high resolution 3D reconstructions of the particles which were then used to generate realistic micromagnetic simulations which can be directly compared to experimental results. Such realistic simulations minimize the amount of challenging experimental work that must be carried out, since a larger range of experimental conditions can be investigated via simulations. The simulations presented here are in good agreement with the MTXM data.

Results of MTXM studies and micromagnetic simulations support previous studies which suggest dusty olivines are extremely reliable paleomagnetic recorders. Fe inclusions exhibiting both single vortex and Kittel closure structures are stable in applied fields of at least $200 \mathrm{mT}$, and simulations suggest they are stable in significantly higher applied fields. This is much stronger than any magnetic field naturally generated within the solar system, suggesting it is unlikely dusty olivines have been overprinted by other magnetic fields after acquiring remanence.

(C)2019 American Geophysical Union. All Rights Reserved. 
This is the first correlative approach resulting in the direct characterization of the behaviour and internal magnetic structure of nanoparticles in the vortex size range. This is relevant for the majority of paleomagnetic carriers, which exhibit vortex to multivortex behaviour. Vortex grains are still poorly understood and essential for furthering the applications of paleomagnetic study, such as unraveling Earth's earliest paleomagnetic record and interpreting extraterrestrial paleomagnetic signals from meteorites.

Acknowledgments. We thank Trevor Almeida and Roger Fu for very helpful review feedback. The research leading to these results has received funding from the European Research Council under the European Union's Seventh Framework Programme (FP/20072013) ERC Grant Agreement No. 320750. Mi-Young Im acknowledges support from DGIST R\&D program of the Ministry of Science, ICT and Future Planning (18-BT02). Work at ALS was supported by the US Department of Energy under contract no. DE-AC02-05CH11231. We thank Roger Fu and Benjamin Weiss for providing us with samples of the Semarkona chondrite. James Bryson and Ioan Lascu assisted with MTXM experiments. Jon Barnard provided advice and assistance for the STEM tomography experiment. Pádraig Ó Conbhuí provided the code to convert 3D micromagnetic simulations to a regular $2 \mathrm{D}$ grid. All data files are available in the supporting information.

\section{References}

Ahrens, J., C. Law, B. Geveci, James Ahrens, and Charles L. B. Geveci, Paraview: An end user tool for large data visualization, The visualization handbook, 836, 717-731, doi:10.1016/B978-012387582-2/50038-1, 2005.

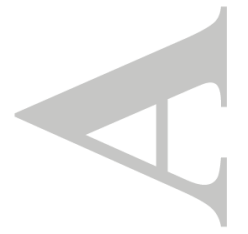

(C)2019 American Geophysical Union. All Rights Reserved. 
Almeida, T. P., T. Kasama, A. R. Muxworthy, W. Williams, L. Nagy, and R. E. Dunin-Borkowski, Observing thermomagnetic stability of nonideal magnetite particles: Good paleomagnetic recorders?, Geophysical Research Letters, 41(20), 7041-7047, doi: 10.1002/2014GL061432, 2014.

Almeida, T. P., A. R. Muxworthy, A. Kovacs, W. Williams, L. Nagy, P. Ó. Conbhuí, C. Frandsen, R. Supakulopas, and R. E. Dunin-borkowski, Direct observation of the thermal demagnetization of magnetic vortex structures in nonideal magnetite recorders, Geophysical Research Letters, 43, 8426-8434, doi:10.1002/2016GL070074.Received, 2016.

Ayachit, U., The ParaView Guide, www.paraview.org, 2016.

Chapman, J. N., and M. R. Scheinfein, Transmission electron microscopies of magnetic microstructures, Journal of Magnetism and Magnetic Materials, 200(1), 729-740, doi: 10.1016/S0304-8853(99)00317-0, 1999.

Choe, S.-B., Y. Acremann, A. Scholl, A. Bauer, A. Doran, J. Stöhr, and H. A. Padmore, Vortex Core - Driven Magnetization Dynamics, Science, 304, 420-423, 2004.

Conbhuí, P. Ó., W. Williams, and L. Nagy, Simulated electron holography of PSD particles, EGU General Assembly, 18(18534), 2016.

Conbhuí, P. Ó., W. Williams, K. Fabian, P. Ridley, L. Nagy, and A. R. Muxworthy, MERRILL: Micromagnetic Earth Related Robust Interpreted Language Laboratory, Geochemistry, Geophysics, Geosystems, pp. 1-27, doi:10.1002/2017GC007279, 2018.

Donnelly, C., M. Guizar-Sicairos, V. Scagnoli, S. Gliga, M. Holler, J. Raabe, and L. J. Heyderman, Three-dimensional magnetization structures revealed with X-ray vector nanotomography, Nature, 547(7663), 328-331, doi:10.1038/nature23006, 2017.

(C)2019 American Geophysical Union. All Rights Reserved. 
Einsle, J. F., et al., Multi-scale three-dimensional characterization of iron particles in dusty olivine: Implications for paleomagnetism of chondritic meteorites, American Mineralogist, 101, 2070-2084, doi:10.2138/am.2010.3521, 2016.

Falch, K. V., D. Casari, M. Di Michiel, C. Detlefs, A. Snigireva, I. Snigireva, V. Honkimäki, and R. H. Mathiesen, In situ hard X-ray transmission microscopy for material science, Journal of Materials Science, 52(6), 3497-3507, doi:10.1007/s10853-016-0643-8, 2017.

Fischer, P., Eimuller T., G. Schutz, M. Kohler, G. Bayreuther, G. Denbeaux, and D. Attwood, Study of in-plane magnetic domains with magnetic transmission x-ray microscopy, Journal of Applied Physics, 89(11), 7159-7161, doi:10.1063/1.1355333, 2001.

Fischer, P., D.-H. Kim, W. Chao, J. A. Liddle, E. H. Anderson, and D. T. Attwood, Soft X-ray microscopy of nanomagnetism, Materials Today, 9(1-2), 26-33, doi:10.1016/ S1369-7021(05)71335-3, 2006.

Fu, R. R., et al., Solar nebula magnetic fields recorded in the Semarkona meteorite, Science, 346, 1-9, 2014a.

Fu, R. R., et al., Solar nebula magnetic fields recorded in the Semarkona meteorite, Science, 346(6213), 12, 2014b.

Guslienko, K. Y., X. F. Han, D. J. Keavney, R. Divan, and S. D. Bader, Magnetic vortex core dynamics in cylindrical ferromagnetic dots, Physical Review Letters, 96 (6), 1-4, doi:10.1103/PhysRevLett.96.067205, 2006.

Im, M. Y., K. S. Lee, A. Vogel, J. I. Hong, G. Meier, and P. Fischer, Stochastic formation of magnetic vortex structures in asymmetric disks triggered by chaotic dynamics, Nature Communications, 5, 1-6, doi:10.1038/ncomms6620, 2014.

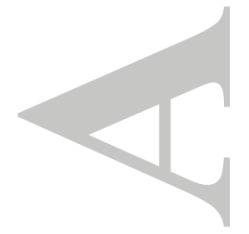

(C)2019 American Geophysical Union. All Rights Reserved. 
Kasai, S., P. Fischer, M. Y. Im, K. Yamada, Y. Nakatani, K. Kobayashi, H. Kohno, and T. Ono, Probing the Spin polarization of current by soft X-ray imaging of currentinduced magnetic vortex dynamics, Physical Review Letters, 101 (23), 1-4, doi:10.1103/ PhysRevLett.101.237203, 2008.

Kasama, T., R. E. Dunin-Borkowski, T. Asaka, R. J. Harrison, R. K. Chong, S. A. Mcenroe, E. T. Simpson, Y. Matsui, and A. W. Putnis, The application of Lorentz transmission electron microscopy to the study of lamellar magnetism in hematite-ilmenite, American Mineralogist, 94(2-3), 262-269, doi:10.2138/am.2009.2989, 2009.

Kittel, C., Theory of the structure of ferromagnetic domains in films and small particles, Physical Review, 70(11-12), 965-971, doi:10.1103/PhysRev.70.965, 1946.

Lappe, S. C. L. L., N. S. Church, T. Kasama, A. B. Da Silva Fanta, G. Bromiley, R. E. Dunin-Borkowski, J. M. Feinberg, S. Russell, and R. J. Harrison, Mineral magnetism of dusty olivine: A credible recorder of pre-accretionary remanence, Geochemistry, Geophysics, Geosystems, 12, 1-20, doi:10.1029/2011GC003811, 2011.

Lascu, I., J. F. Einsle, M. R. Ball, and R. J. Harrison, The Vortex State in Geologic Materials: A Micromagnetic Perspective, Journal of Geophysical Research: Solid Earth, 123(9), 7285-7304, doi:10.1029/2018JB015909, 2018.

Leary, R., Z. Saghi, P. A. Midgley, and D. J. Holland, Compressed sensing electron tomography, Ultramicroscopy, 131, 70-91, doi:10.1016/j.ultramic.2013.03.019, 2013.

Leroux, H., G. Libourel, L. Lemelle, and F. Guyot, Experimental study and TEM characterization of dusty olivines in chondrites: Evidence for formation by in situ reduction, Meteoritics $\&$ Planetary Science, 38(1), 81-94, doi:10.1111/j.1945-5100.2003.tb01047.x, 2003.

(C)2019 American Geophysical Union. All Rights Reserved. 
Midgley, P. A., and R. E. Dunin-Borkowski, Electron tomography and holography in materials science, Nature Materials, 8(4), 271-280, doi:10.1038/nmat2406, 2009.

Midgley, P. A., and M. Weyland, 3D electron microscopy in the physical sciences: The development of Z-contrast and EFTEM tomography, Ultramicroscopy, 96(3-4), 413431, doi:10.1016/S0304-3991(03)00105-0, 2003.

Muxworthy, A. R., and W. Williams, Critical single-domain grain sizes in elongated iron particles: implications for meteoritic and lunar magnetism, Geophysical Journal International, 202(1), 578-583, doi:10.1093/gji/ggv180, 2015.

Nagy, L., W. Williams, A. R. Muxworthy, K. Fabian, T. P. Almeida, O. Conbhuí, and V. P. Shcherbakov, Stability of equidimensional pseudosingle-domain magnetite over billion-year timescales, Proceedings of the National Academy of Sciences, doi:10.1073/ pnas.1708344114, 2017.

Nagy, L., W. Williams, L. Tauxe, A. R. Muxworthy, and I. Ferreira, Thermomagnetic recording fidelity of nanometer-sized iron and implications for planetary magnetism, Proceedings of the National Academy of Sciences, p. 201810797, doi:10.1073/pnas. 1810797116, 2019.

Raabe, J., C. Quitmann, C. H. Back, F. Nolting, S. Johnson, and C. Buehler, Quantitative analysis of magnetic excitations in landau flux-closure structures using synchrotronradiation microscopy, Physical Review Letters, 94(21), 1-4, doi:10.1103/PhysRevLett. 94.217204, 2005.

Roberts, A. P., L. Tauxe, D. Heslop, X. Zhao, and Z. Jiang, A Critical Appraisal of the Day' Diagram, Journal of Geophysical Research: Solid Earth, pp. 2618-2644, doi: 10.1002/2017JB015247, 2018.

(C)2019 American Geophysical Union. All Rights Reserved. 
Saghi, Z., D. J. Holland, R. Leary, A. Falqui, G. Bertoni, A. J. Sederman, L. F. Gladden, and P. A. Midgley, Three-dimensional morphology of iron oxide nanoparticles with reactive concave surfaces. A compressed sensing-electron tomography (CS-ET) approach, Nano Letters, 11(11), 4666-4673, doi:10.1021/nl202253a, 2011.

Schaffer, M., B. Schaffer, and Q. Ramasse, Sample preparation for atomic-resolution STEM at low voltages by FIB, Ultramicroscopy, 114, 62-71, doi:10.1016/j.ultramic. 2012.01.005, 2012.

Shaar, R., and L. Tauxe, Thellier GUI: An integrated tool for analyzing paleointensity data from Thellier-type experiments, Geochemistry, Geophysics, Geosystems, 14(3), 677-692, doi:10.1002/ggge.20062, 2013.

Shah, J., et al., The oldest magnetic record in our solar system identified using nanometric imaging and numerical modeling, Nature Communications, 9(1), 9-14, doi:10.1038/ s41467-018-03613-1, 2018.

Uehara, M., and N. Nakamura, Experimental constraints on magnetic stability of chondrules and the paleomagnetic significance of dusty olivines, Earth and Planetary Science Letters, 250, 292-305, doi:10.1016/j.epsl.2006.07.042, 2006.

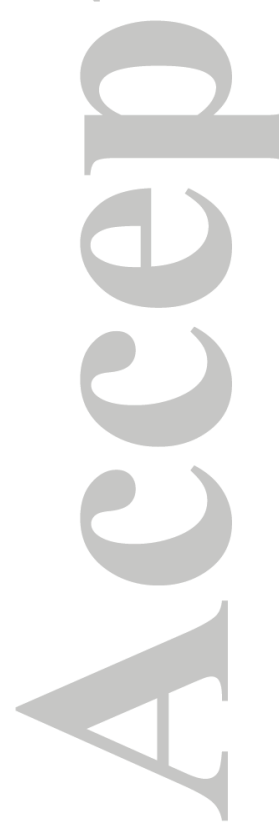

(C)2019 American Geophysical Union. All Rights Reserved. 
Table 1. Dimensions of the studied Fe particles within a dusty olivine grain.

\begin{tabular}{lccc} 
Particle & Height $(\mathrm{nm})$ & Major Axis $(\mathrm{nm})$ & Minor Axis $(\mathrm{nm})$ \\
\hline Particle 1 & 200 & 345 & 250 \\
Particle 2 & 200 & 245 & 220 \\
Particle 3 & 200 & 330 & 365 \\
Particle 4 & 250 & 605 & 455 \\
Particle 5 & 200 & 525 & 455 \\
\hline
\end{tabular}

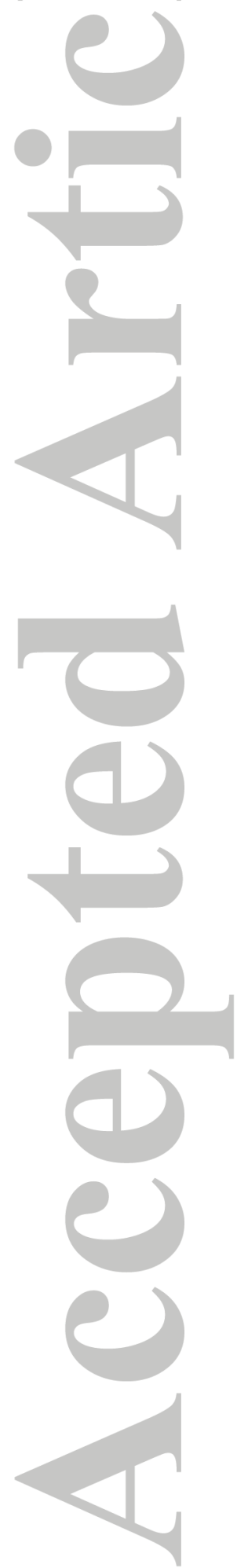

(C)2019 American Geophysical Union. All Rights Reserved. 
Table 2. Applied field strengths leading to annihilation of vortices from micromagnetic simulations

\begin{tabular}{llcccc} 
& \multicolumn{5}{c}{ Applied Field (mT) } \\
Particle & OOP & IP & OOP1 & OOP2 \\
\hline Particle 1 & - & 370 & 370 & 510 \\
Particle 2 & - & 550 & 490 & 480 \\
Particle 3 & - & 330 & 330 & 490 \\
Particle 4 & 780 & - & - & - \\
Particle 5 & $>1000$ & - & - & - \\
\hline
\end{tabular}

(C)2019 American Geophysical Union. All Rights Reserved. 

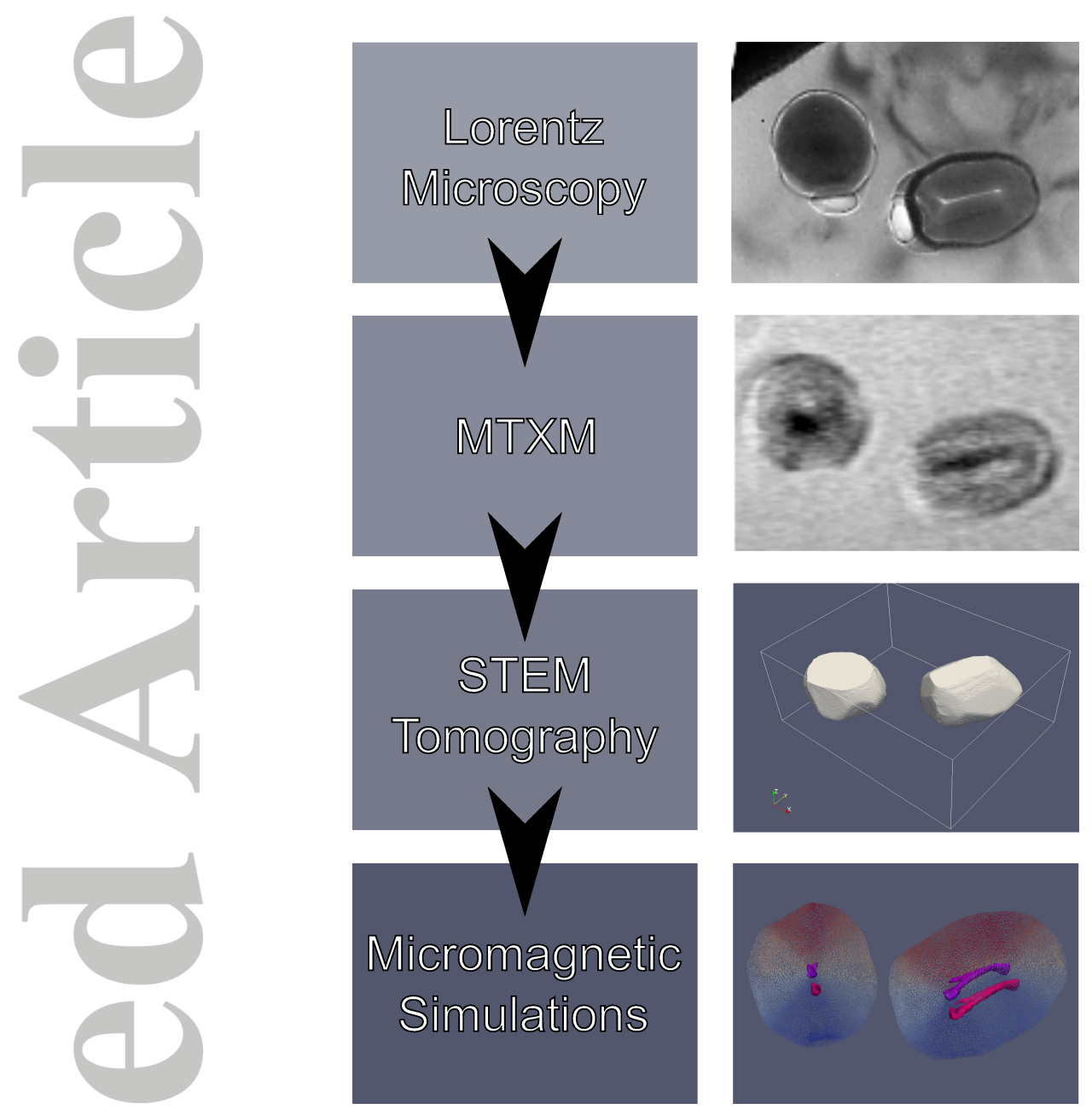

Figure 1. A correlative approach is demonstrated in order to investigate the fundamental properties of nanoscale magnetic particles. Results of Lorentz microscopy are combined with results on the same particles using transmission X-ray microscopy (MTXM). A 3D reconstruction of the imaged particles is then generated using STEM tomography. These 3D reconstructions are used to carry out realistic micromagnetic simulations using MERRILL. In the micromagnetic image shown, the core positions for positive and negative applied fields (pink and purple, respectively) are both shown.

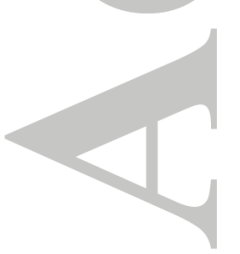

(C)2019 American Geophysical Union. All Rights Reserved. 


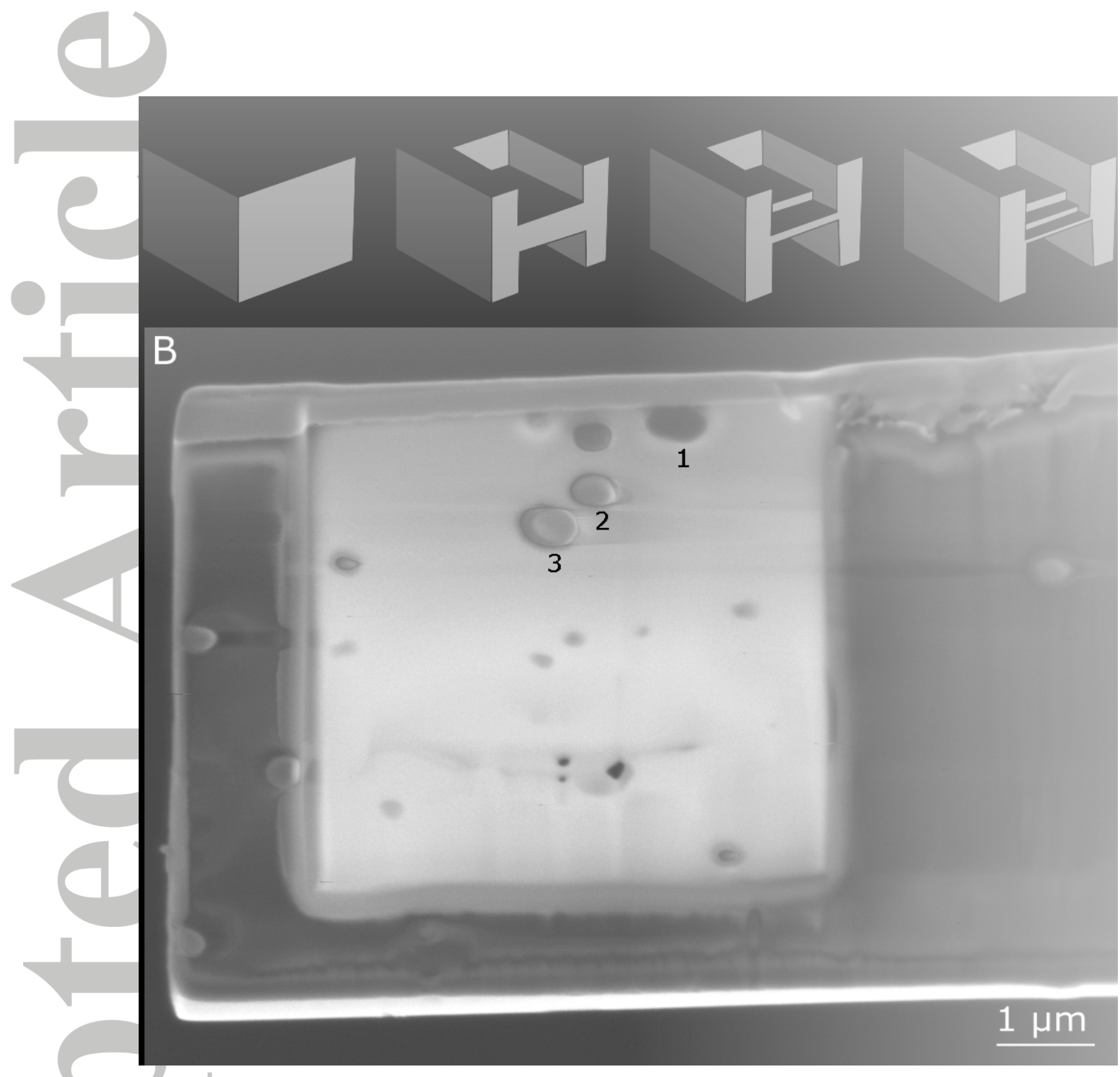

Figure 2. (a) The schematic shows the adaptation of the FIB preparation method [Schaffer et al., 2012] used in this study, where a stable window configuration is made to stabilize the extremely thin region of the sample imaged using TXM. (b) An SEM image of the prepared stable window configuration for the FIB lamellae containing Particles 1,2 and 3.

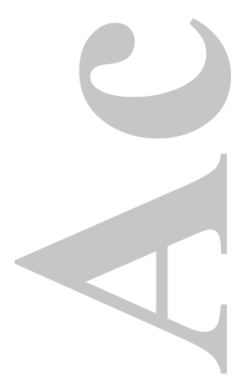

(C)2019 American Geophysical Union. All Rights Reserved. 

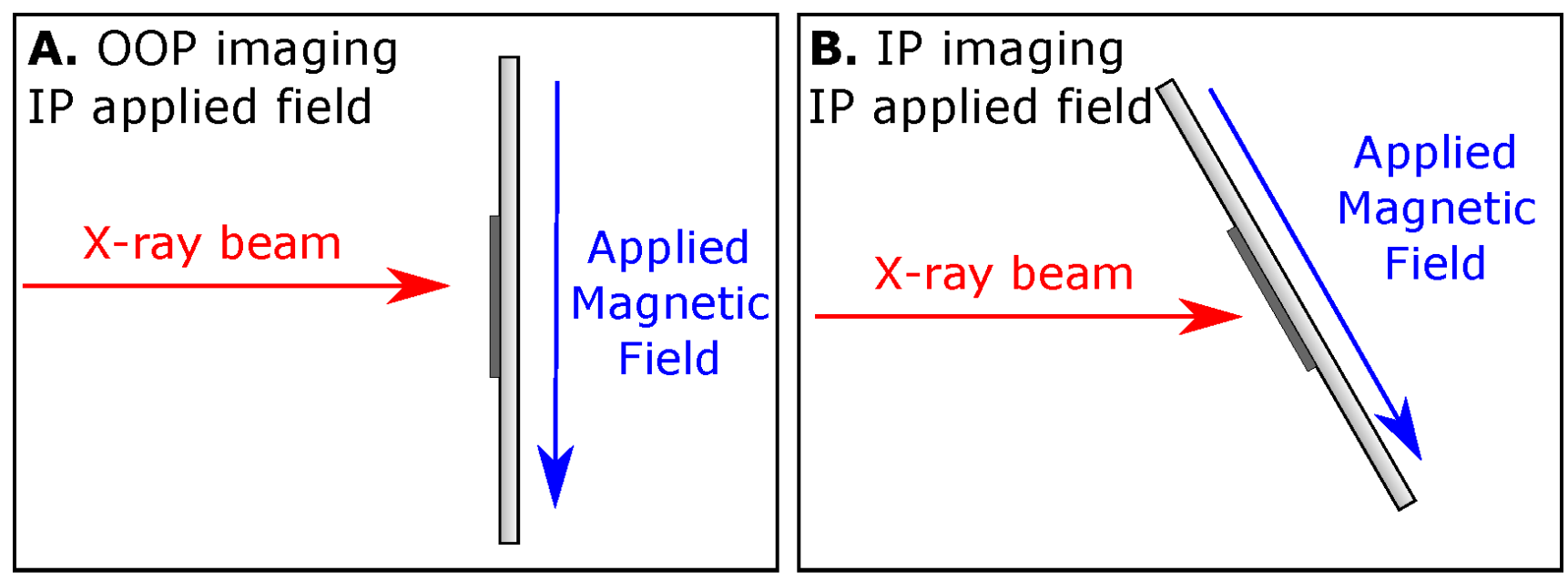

\section{OOP imaging OOP applied field}

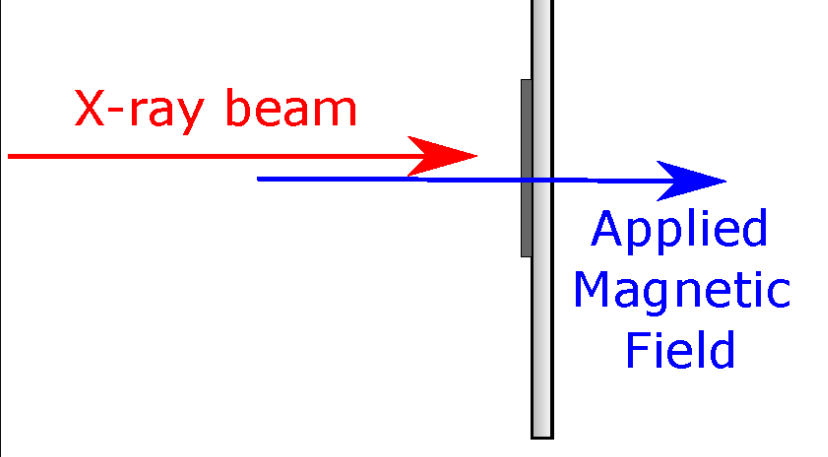

D. IP imaging OOP applied field

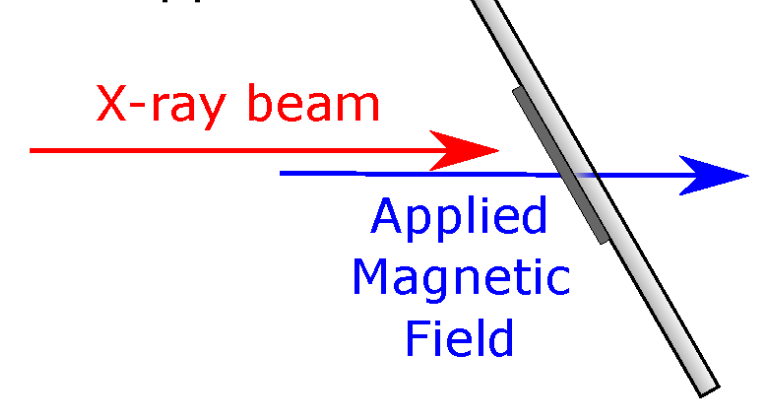

Figure 3. Schematic showing the four experimental configurations in which images were acquired. (a) OOP imaging where the X-ray beam is perpendicular to the sample surface, and the IP magnetic field is parallel to the sample surface. (b) IP imaging where the X-ray beam is at an angle of $60^{\circ}$ to the sample surface and the applied magnetic field is parallel to the sample surface. (c) OOP imaging and OOP magnetic field; both are perpendicular to the sample surface. (d) IP imaging and OOP magnetic field, where both the X-ray beam and applied magnetic field are at $60^{\circ}$ to the sample surface.

(C)2019 American Geophysical Union. All Rights Reserved. 

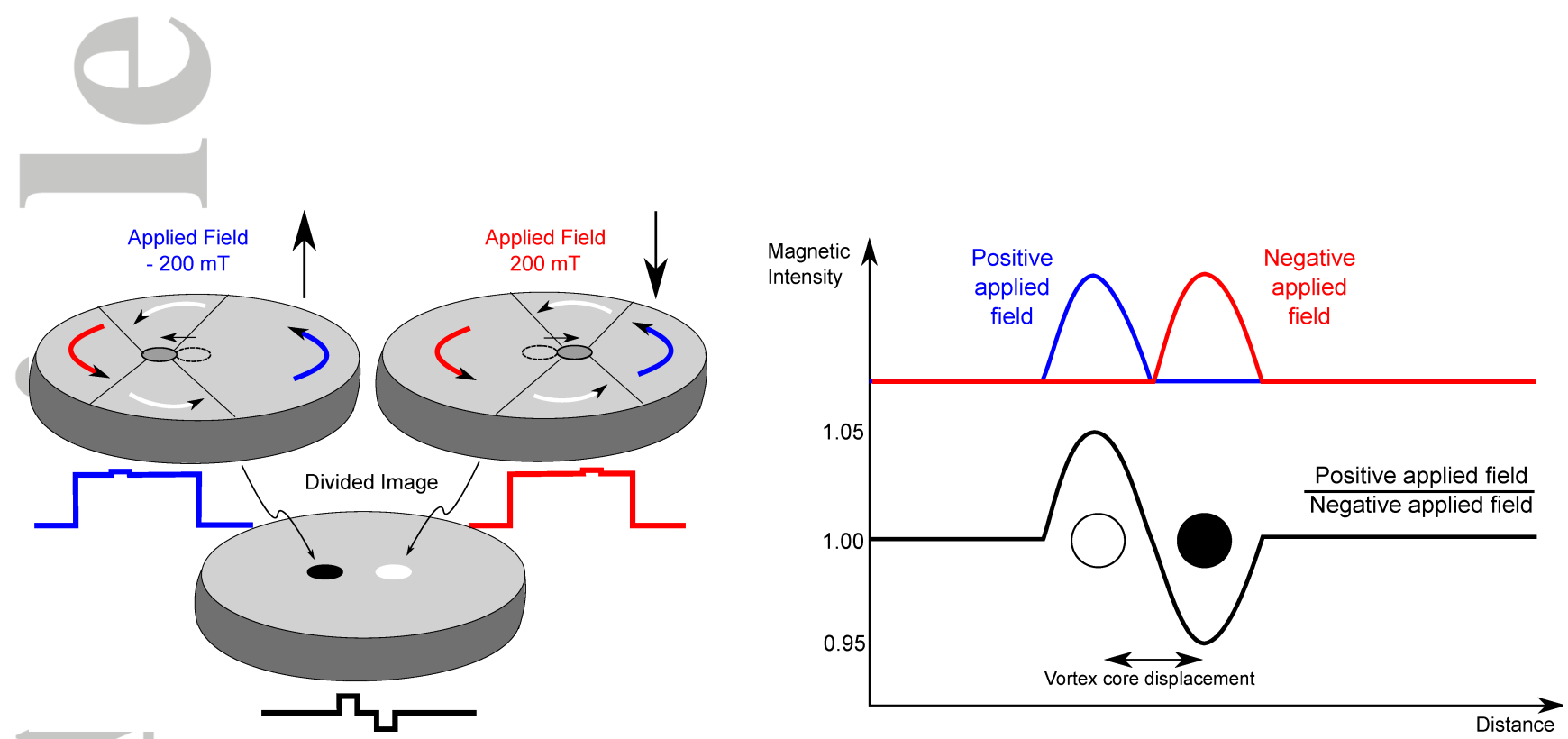

Figure 4. Schematics showing how images are divided to generate magnetic contrast. Negative and positive applied fields are colored blue and red, respectively. In the left-hand cartoon, the profiles show an idealized absorption signal, which is dominated by the Fe particle, with the small magnetic signal superimposed on the large absorption. Opposite applied fields cause opposing shifts in the position of the vortex core. The position of the vortex core is associated with a peak in X-ray absorption, which can be considered to relate directly to magnetic intensity. By dividing images with opposite vortex core shifts (shown in the right-hand figure), a bright and dark spot are generated, showing the relative position of the vortex core under the two applied fields and removing the large absorption peak generated by the Fe particle. 


\section{Applied Field $+1 \mathrm{~A}$}

One image:

Applied Field

$-1 A$

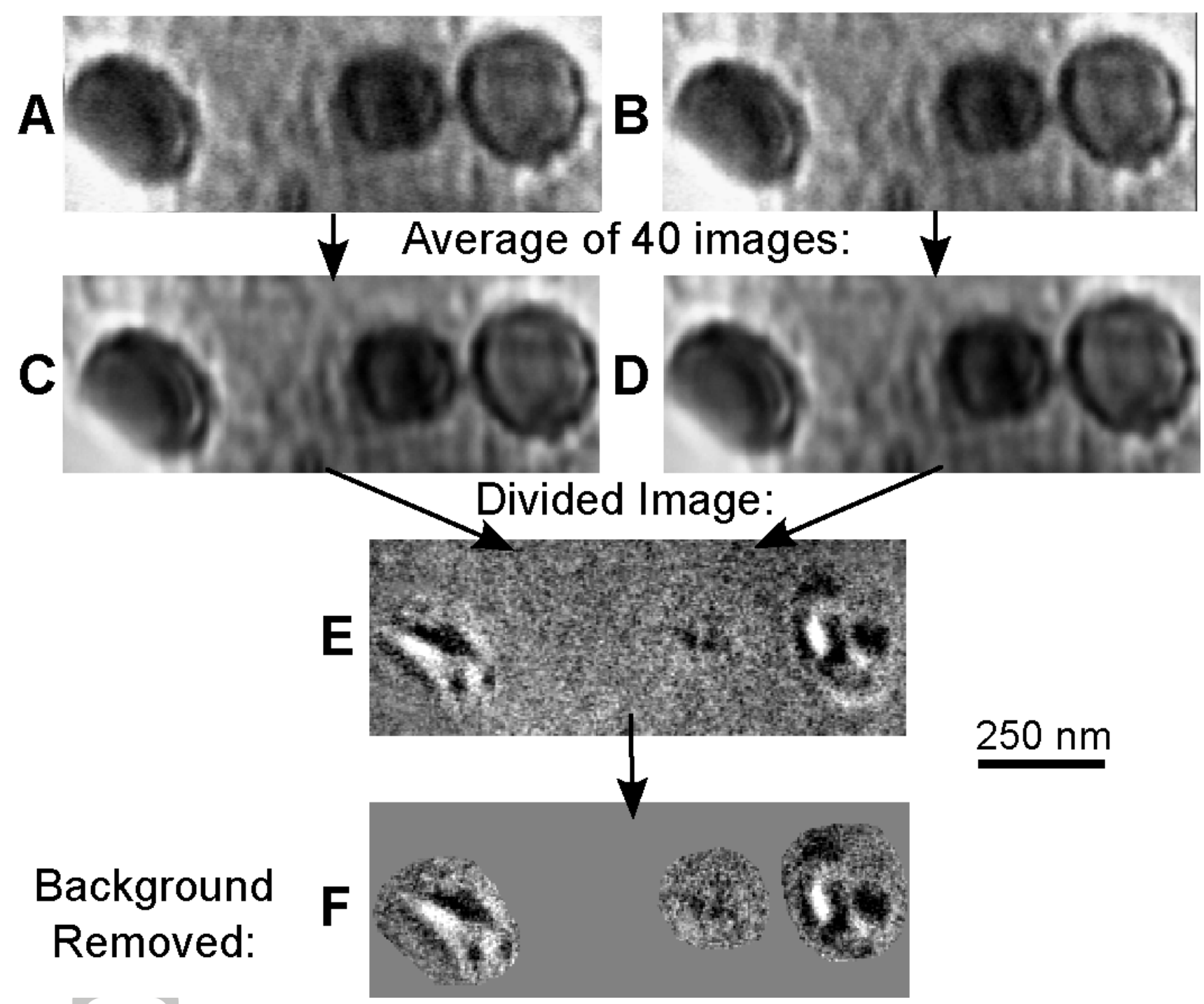

Figure 5. (a) Particles are imaged under an applied field of $1 \mathrm{~A}(\sim 200 \mathrm{mT})$. (b) Particles are imaged again under a 1 A applied field in the opposite direction. (c) and (d) 40 images are acquired of (a) and (b) respectively, which are then aligned and averaged. (e) Images (c) and (d) are divided by each other to remove the strong absorption of the Fe particle and reveal the magnetic contrast. (f) The background is then manually removed so that only the contrast within the particles is observed.

(C)2019 American Geophysical Union. All Rights Reserved. 

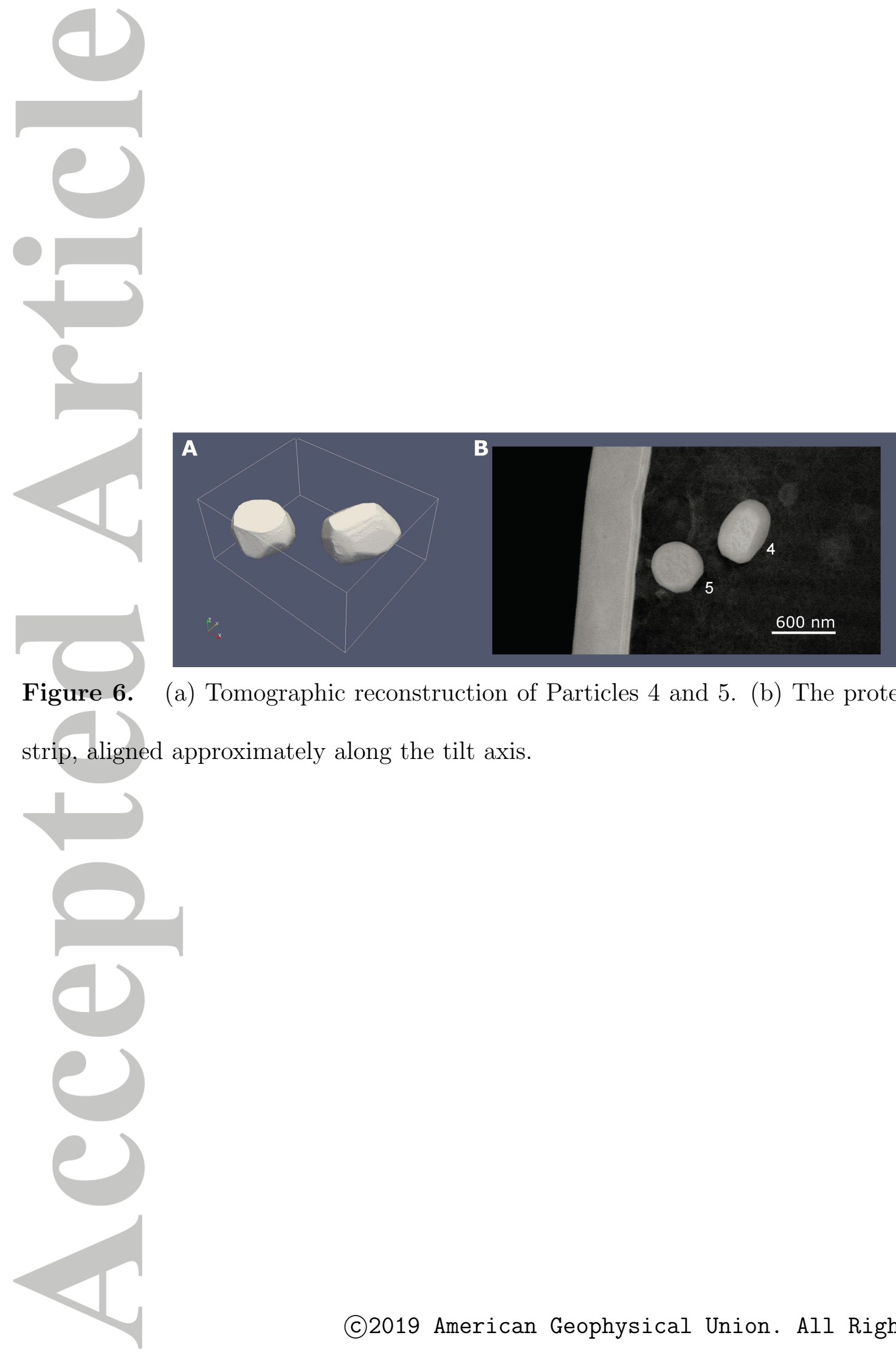

Figure 6. (a) Tomographic reconstruction of Particles 4 and 5. (b) The protective platinum strip, aligned approximately along the tilt axis.

(C)2019 American Geophysical Union. All Rights Reserved. 


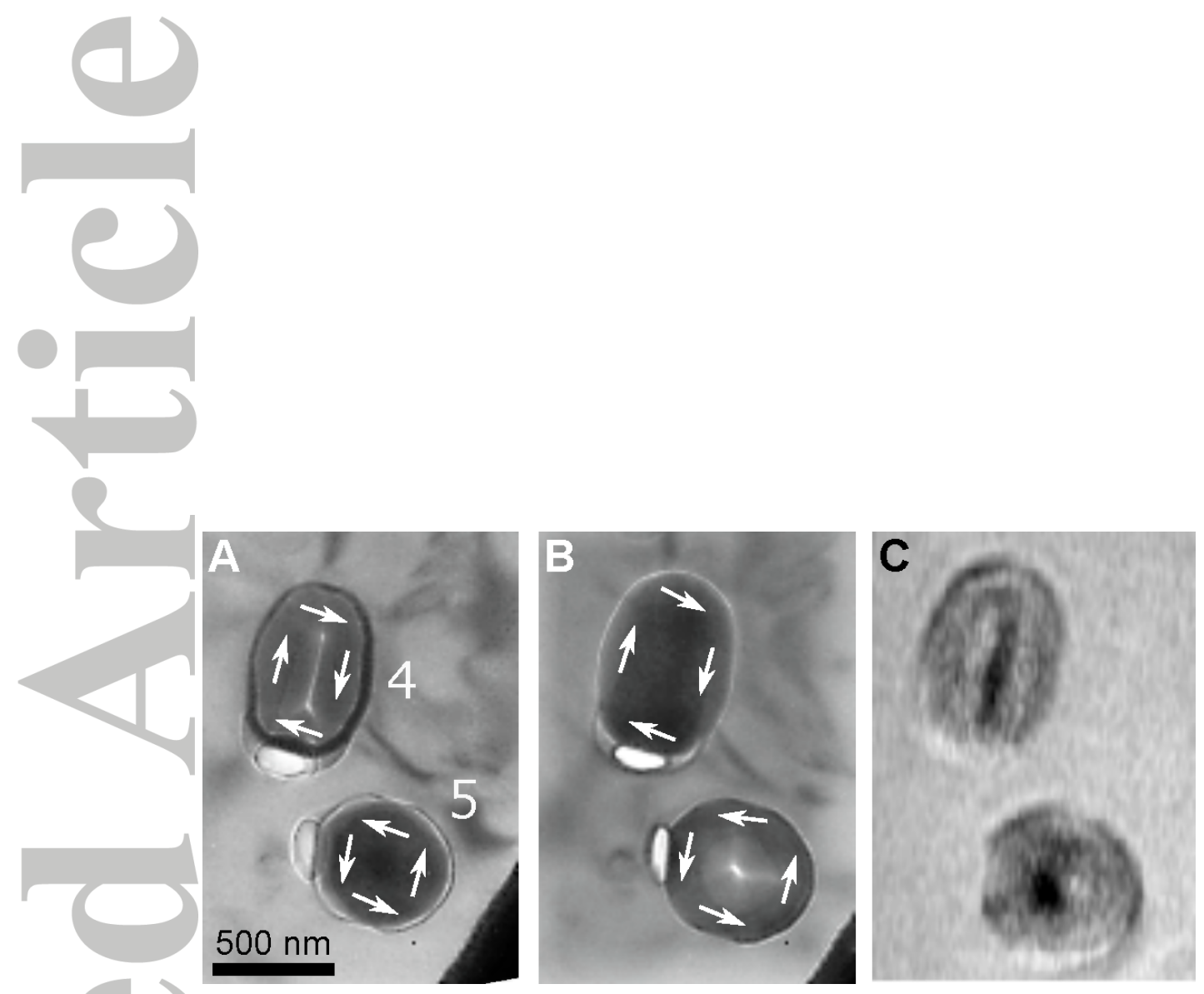

Figure 7. a) An over-focused Lorentz image (b) An under-focused Lorentz image (c) A MTXM image. Similar contrast is observed in both particles.

(C)2019 American Geophysical Union. All Rights Reserved. 

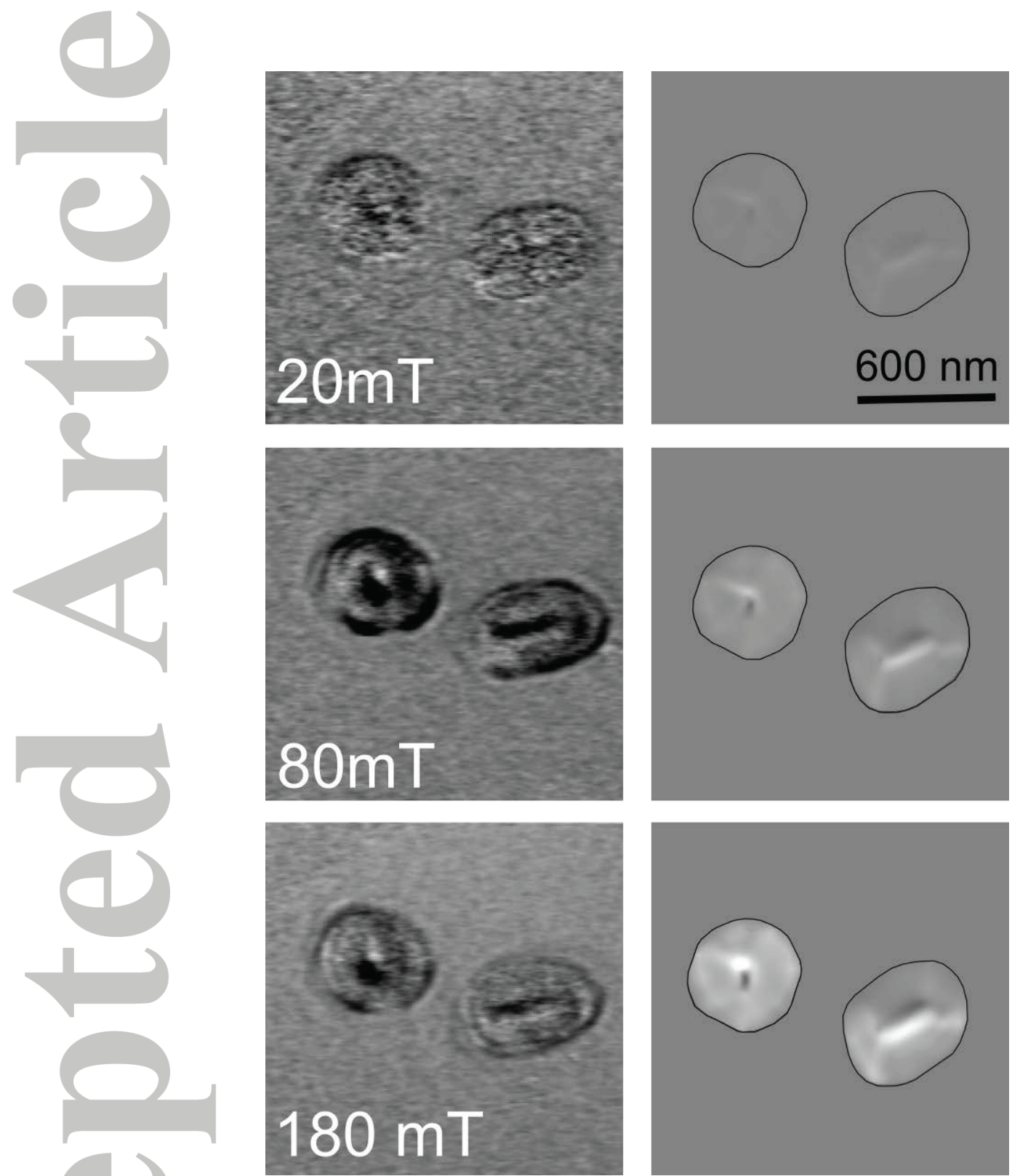

Figure 8. The MTXM images are in good agreement with simulations for the same projection. The grey background has a uniform value of 1, i.e., there is no difference between the divided images in these regions. Background noise has been removed.

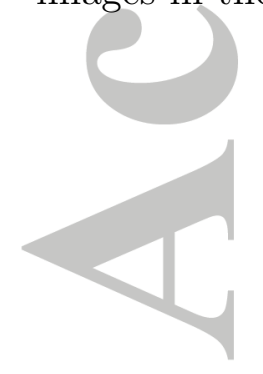

(C)2019 American Geophysical Union. All Rights Reserved. 

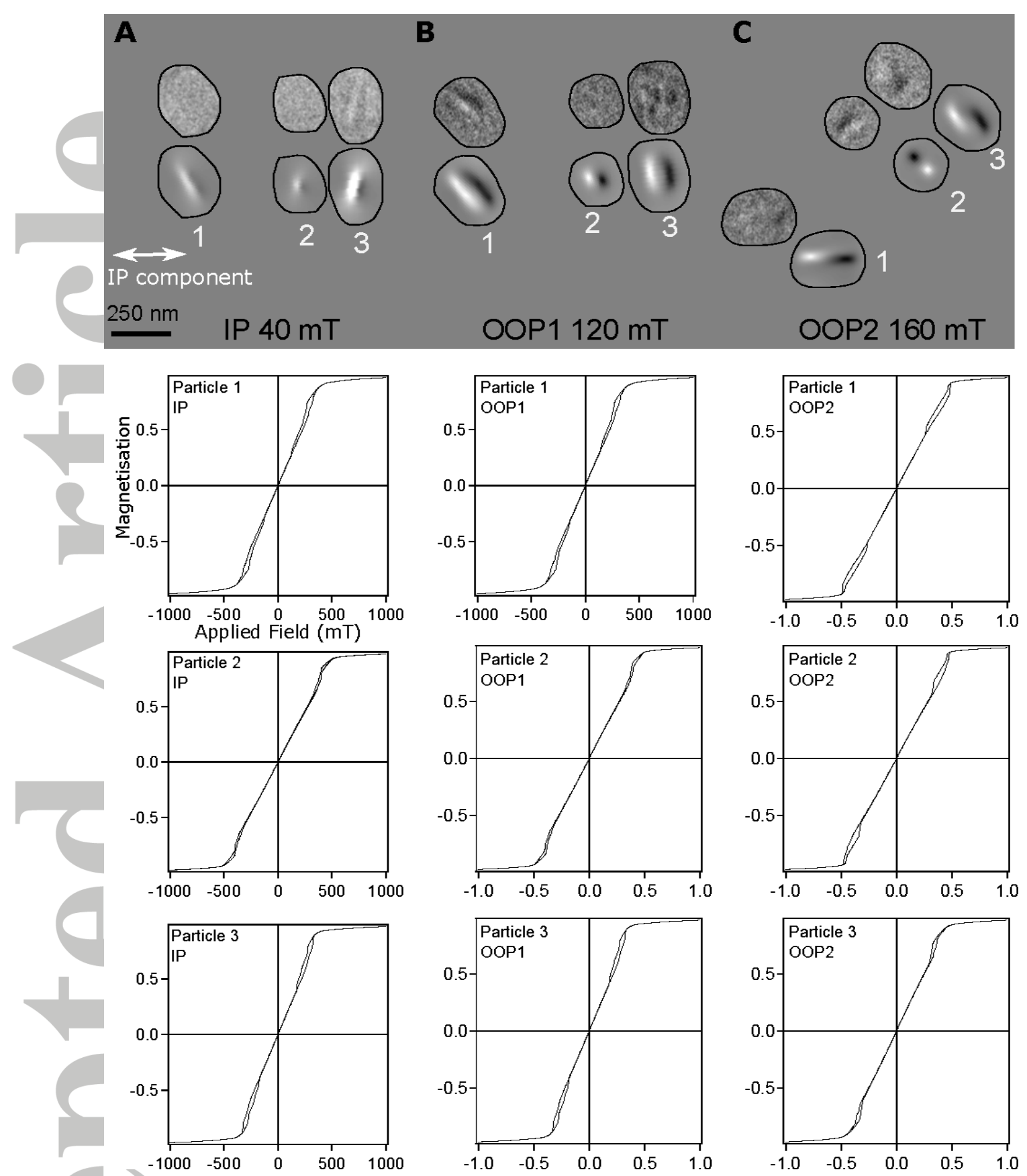

Figure 9. Examples of MTXM images and corresponding simulations are shown for three more particles in a dusty olivine from the Semarkona chondrite. Hysteresis loops for the three applied field configurations were calculated using micromagnetic simulations $( \pm 1 \mathrm{~T})$. (a) In-plane applied field. (b) An out-of-plane applied field (orientation 1). (c) The second orientation (orientation 2) for an out-of-plane applied field. The change in orientation of the particles reflects how the particles were rotated relative to the X-ray beam.

(C)2019 American Geophysical Union. All Rights Reserved. 


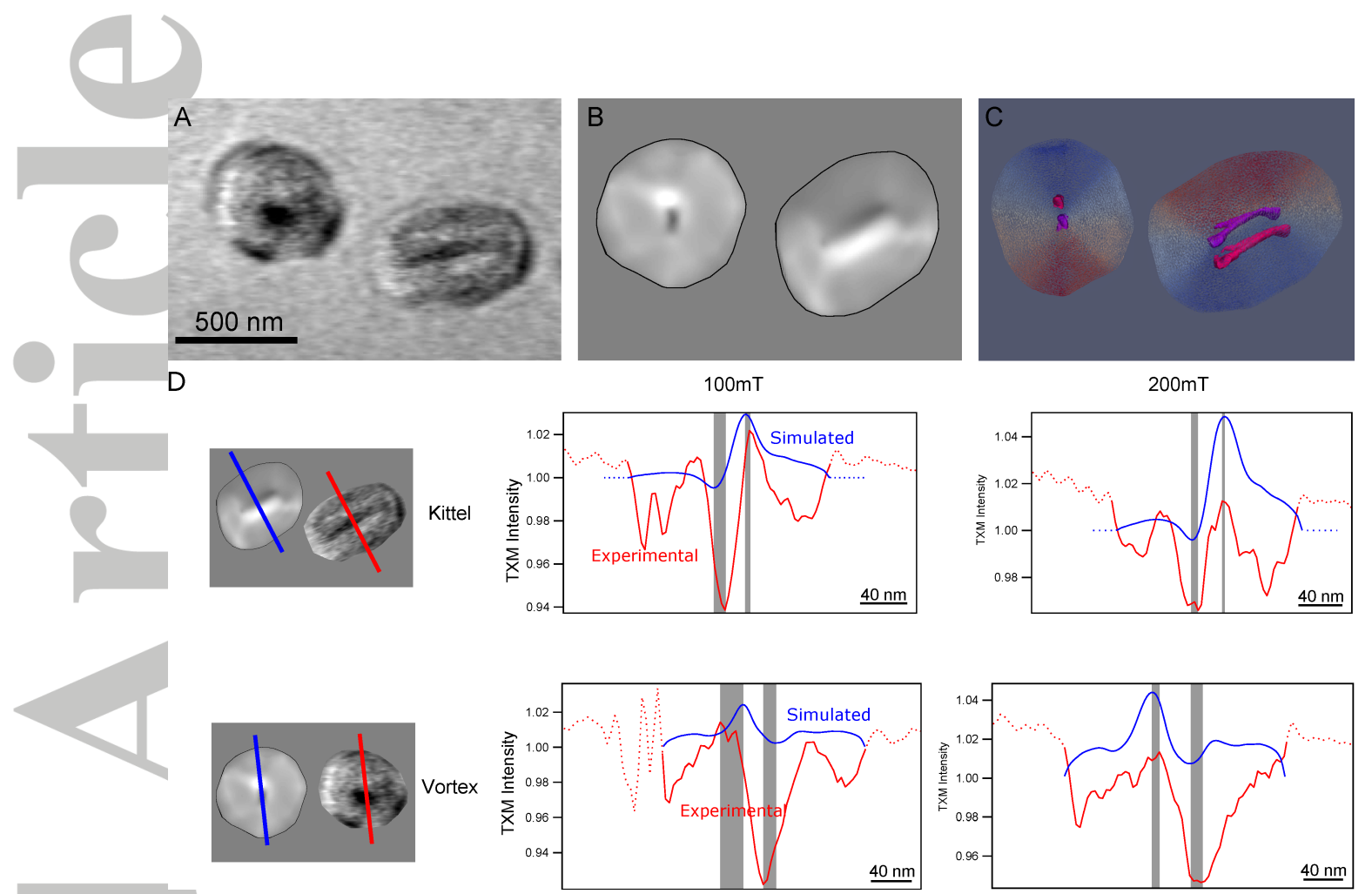

Figure 10. (a) A MTXM image for a $200 \mathrm{mT}$ OOP applied field. (b) The corresponding simulated MTXM image, from the micromagnetic simulations. (c) A 3D visualization in Paraview of the actual change in magnetic structure in response to an applied field. The purple and pink regions are selected as lines of constant moment and correspond to an applied field of - $200 \mathrm{mT}$ and $200 \mathrm{mT}$ respectively. (d) Red lines represent profiles across MTXM images and blue lines represent profiles across simulated images. Grey bars show the misfit between simulated and experimental peaks and troughs, which represent the offset in magnetic structure for a given applied field. The amplitude of the contrast is on an arbitrary scale and cannot be directly compared between simulated and MTXM data.

(C)2019 American Geophysical Union. All Rights Reserved. 